\title{
Pion gas viscosity at low temperature and density
}

\author{
Antonio Dobado* \\ Departamento de Física Teórica, Universidad Complutense de Madrid, 28040 Madrid, Spain \\ Silvia N. Santalla ${ }^{\dagger}$ \\ Departamento de Física, Universidad Carlos III de Madrid, 28911 Leganés, Spain
}

(Received 24 December 2001; published 15 May 2002)

\begin{abstract}
By using chiral perturbation theory and the Uehling-Uhlenbeck equation we compute the viscosity of a pion gas, in the low temperature and low density regime, in terms of the temperature and the pion fugacity. The viscosity turns out to be proportional to the square root of the temperature over the pion mass. Next to leading corrections are proportional to the temperature over the pion mass to $3 / 2$.
\end{abstract}

DOI: 10.1103/PhysRevD.65.096011

\section{INTRODUCTION}

The possibility of discovering the so-called quark-gluon plasma (QGP) at BNL RHIC (Relativistic Heavy Ion Collider) or in the future CERN LHC (Large Hadron Collider) has triggered a lot of effort on the theoretical description of ultrarelativistic heavy ion collisions. The modern view of these collisions was set mainly by Bjorken [1] and it is based on the hydrodynamic model already considered by Landau [2]. In the last stages of the evolution of the central rapidity region the hadronic fluid is made mainly of pions. It has been argued that this expanding pion gas can reach thermal equilibrium much more efficiently than chemical equilibrium since at low energies pion interactions are mostly elastic [3]. In order to have an appropriate phenomenological description of the expanding pion gas it is needed to set the proper hydrodynamic equations. In the pioneering work by Bjorken the hadronic gas (both in the confined and in the quark-gluon plasma phases) was assumed to be a perfect relativistic fluid and accordingly viscosity and heat conductivity were neglected. In order to check the validity of this assumption in the different regimes of the pion gas expansion we need to compute the corresponding transport coefficients as a function of the temperature and density. This could make it possible to know in what physical situations they are relevant and the ideal fluid equations are not appropriate anymore. In addition the expressions obtained for these coefficients can be used to set the correct relativistic version of the NavierStokes equations which applies when dissipative effects must be taken into account in the pion fluid dynamics. Curiously enough, the computation of these coefficients is also interesting from the fundamental point of view, since it can be done completely from first principles.

In this work we will illustrate this by computing the viscosity of the pion gas at low energy and density. Our computation will rely just on standard kinetic theory and the chiral symmetry properties of the strong interaction and thus our results can be considered model independent. In fact our results will depend only on the pion mass $M_{\pi}$ and the pion decay constant $F_{\pi}$. The main points on which our computa-

\footnotetext{
*Electronic address: dobado@sagan.gae.ucm.es

†Electronic address: ssantall@ fis.uc3m.es
}

PACS number(s): 11.30.Rd, 12.39.Fe, 24.10.Nz, 25.75.-q

tion is based are the following. First of all our computation is going to apply only at low temperature and density. At low temperatures hadronic matter is confined into hadrons and at very low temperatures the only excited modes are pions for vanishing baryonic number $n_{B}$ density. Notice that $n_{B} \simeq 0$ is supposed to be the case in the central rapidity region of heavy ion collisions. Moreover, the additional low-density condition allows us to consider the nonbaryonic hadronic matter as a pion gas.

At low temperatures most of the pion interactions occur at low energies. The low energy pion interactions are completely determined by the chiral symmetry pattern of the strong interactions. In the chiral limit (vanishing $u$ and $d$ quark masses) the strong interaction dynamics is invariant under the $\mathrm{SU}(2)_{L} \times \mathrm{SU}(2)_{R}$ chiral transformations. However this symmetry is supposed to be spontaneously broken to the diagonal or isospin group $\mathrm{SU}(2)_{L+R}$, the pions being the corresponding Goldstone bosons. In the real world quarks masses are different from zero and then this symmetry breaking pattern is not exact resulting in a small value (when compared with other hadrons) for the pion mass $M_{\pi}$. In any case chiral symmetry determines completely the low energy pion interactions in terms of $M_{\pi}$ and $F_{\pi}$ (Weinberg low energy theorems). The corrections appearing at higher energies can be computed in a systematic way by means of chiral perturbation theory [4]. In the chiral limit the pion scattering amplitude goes to zero in the low energy limit. Out of the chiral limit it is proportional to $M_{\pi}^{2} / F_{\pi}^{2}$. In any case the interaction is small in this regime. Therefore we arrive at the conclusion that nonbaryonic hadronic matter at low temperature and density can be described as a weakly interacting pion gas. Another important observation is that at low temperatures (energies) most of the pion collisions are elastic. This fact implies in particular that the number of pions is conserved. Thus it is possible to introduce the corresponding pion chemical potential $\mu$ (not to be confused with the more usual chemical potential associated to the baryonic number). This makes it possible to consider the pion gas at thermal equilibrium at temperature $T$ for different values of the chemical potential $\mu$, or what it is the same, different pion densities, and not only the case of chemical equilibrium corresponding to $\mu=0$.

According to the previous discussion the simplest state for nonbaryonic hadronic matter at low temperatures and densi- 
ties is just a gas of weakly interacting pions in thermal equilibrium which could be described in a first approximation with a Bose-Einstein distribution. In addition, at very low temperatures the average pion energy is much smaller than the pion mass allowing for to have a nonrelativistic description of the pion gas. As discussed later, the low density condition will prevent the formation of the Bose-Einstein condensate out of the pions, thus making the description of the hadronic matter in the mentioned regime especially simple.

More generally it is possible to consider the case when the equilibrium is only local. In this case the temperature, density (or pion chemical potential), and the overall velocity are space-time functions. In this situation it is possible to have a hydrodynamic description of the system as that considered in the Bjorken picture of heavy ion collisions. A departure from local equilibrium will give rise to dissipative processes like viscosity, which is the main topic of this work. The evolution of the distribution functions outside equilibrium can be studied by using the Uehling-Uhlenbeck equation, which is the quantum version of the Boltzmann transport equation. The computation of the transport coefficients such as viscosity or heat conductivity requires the solution of the transport equation for different specific perturbations of the local equilibrium distribution.

As it was mentioned above, in this work we are interested in the computation of the viscosity of the hadronic fluid with the approximations discussed in the previous paragraphs. In fact it is possible to have a good estimation of this magnitude by neglecting quantum effects. In this case the equilibrium distribution function is just the Boltzmann distribution instead the Bose-Einstein distribution. Moreover the elastic scattering pion cross section is constant at low energies and as we will see it is given by

$$
\sigma=\frac{23}{384} \frac{M_{\pi}^{2}}{\pi F_{\pi}^{4}} .
$$

In addition, at low temperatures we can use the nonrelativistic approximation so that our problem is equivalent to the classical computation of the viscosity of a gas of hard spheres of radius $R$ so that $\sigma=\pi R^{2}$. By using the well known result in this case (see, for instance, [5]) we find the viscosity to be given by

$$
\eta=\frac{1920}{368} \frac{F_{\pi}^{4} \pi^{3 / 2}}{M_{\pi}} \sqrt{\frac{T}{M_{\pi}}} .
$$

This formula provides a nice estimation of the viscosity of the pion gas. However, it does not take into account any quantum effect that can be important at low temperatures. In addition it does not include any dependence in the pion chemical potential, i.e., in the pion density.

In the following we will show how these effects can properly be taken into account to find the pion gas viscosity in terms of the temperature and chemical potential (or density) in the low temperature and density regime at the leading order. We will also estimate the magnitude of the next to leading corrections.
The transport coefficients for the hadronic matter have been considered several times in the literature. In particular in [6] it is possible to find a lot of detailed computations concerning these coefficients and also relaxation times in the confined phase. In the references quoted therein the reader can also find previous computations of the transport coefficients for the QGP phase. In the very complete analysis of [6], kaon and nucleon effects are also included and they consider both chiral and experimental phase shifts. Their work is based on relativistic coupled transport equations in the nondegenerate limit and it is appropriate for moderate and relatively high temperatures. As mentioned before in the work presented here we are interested in the very low temperature regime where the nonrelativistic limit holds and, in addition, quantum effects cannot be neglected any more since they are essential. For this reason we will use the Uehling-Uhlenbeck equation as the transport equation, which takes into account degeration effects, instead of the Boltzmann-like equation considered in [6]. Therefore that work and that shown in this paper can be considered in some sense as complementary.

The plan of the paper goes as follows. In Sec. II we review the main equations of the nonrelativistic ideal pion gas to set the notation and for further reference. In Sec. III we describe briefly the fundamentals of the kinetic theory needed to follow the present work and in Sec. IV we show how the hydrodynamic equations emerge from it. In Sec. V we obtain the equations for the transport coefficients in terms of the appropriate solutions of the linearized transport equations. In Sec. VI we simplify these equations to write them in useful form for finding explicit formulas for the viscosity. In Sec. VII we give the cross section for the low energy elastic pion scattering obtained from the lowest order chiral perturbation theory. In Sec. VIII we obtain the lowest order terms of the pion gas viscosity. In Sec. IX we show our numerical results and discuss the magnitude of the next to leading corrections and in Sec. X we set the main conclusions of our work. Finally in the Appendix we study the properties of the polynomials introduced in this work that play a role similar to the standard Sonine polynomials in the more familiar classic computations.

\section{STATE EQUATION}

In this section we review briefly the equation of state of a free nonrelativistic bosonic gas, which will provide our description of the pion gas at low temperature and density. As it is well known [7], for a large volume the pressure can be written as

$$
P=-\frac{g T}{2 \pi^{2}} \int_{0}^{\infty} d p p^{2} \log \left[1-e^{\beta[\mu-E(p)]}\right]-\frac{g T}{V} \log \left[1-e^{\beta \mu}\right],
$$

where $g$ is the number of pions $(g=3), T=1 / \beta$ is the temperature, $V$ is the volume, $\mu$ is the pion chemical potential, and $E(p)$ is the energy of a nonrelativistic pion in terms of the momentum, i.e., $\vec{p}=M_{\pi} \vec{v}, E(p)=p^{2} / 2 M_{\pi}$. The number density or the number of pions per unit of volume is given by 


$$
n=\frac{g}{2 \pi^{2}} \int_{0}^{\infty} d p p^{2} \frac{1}{e^{-\beta[\mu-E(p)]}-1}+\frac{g}{V} \frac{1}{e^{-\beta \mu}-1},
$$

where the last term is the number of pions with zero momentum per unit of volume. When this term is not negligible it is responsible for the Bose-Einstein condensate. We thus define

$$
n_{0}=\frac{g}{V} \frac{1}{e^{-\beta \mu}-1} .
$$

Clearly the above equations make sense only when the chemical potential satisfies $\mu \leqslant 0$. As a consequence, in the thermodynamic limit, where $N, V \rightarrow \infty$ with $N / V$ constant, we find two phases: In the first one (normal phase) $\mu \leqslant 0$ and $n_{0}=0$. However, if we lower the temperature, keeping a fixed density, $\mu$ increases until $\mu=0$. At that point $n_{0} \neq 0$ and the ground state density starts to grow forming the BoseEinstein condensate. Eventually, at $T=0$, all pions are in the ground state so that $n=n_{0}$. The critical temperature $T_{c}$ where the phase transition occurs can be found to be

$$
T_{c} \equiv \frac{2 \pi}{M_{\pi}}\left(\frac{n}{g \zeta_{3 / 2}}\right)^{2 / 3},
$$

where $\zeta_{3 / 2}$ is the Riemann $\zeta$ function evaluated at $3 / 2$. Therefore the critical temperature can be made arbitrarily small by lowering the density. In this work we are interested in the low temperature and the low density regime. In order to simplify the analysis we will not consider the contribution of the condensate. Thus for a given temperature $T$, we will always assume that the density will be low enough so that $T>T_{c}$ and no significant fraction of pions will have zero momentum (see [8] for a discussion of the pion condensate).

In the following it will also be convenient to introduce the fugacity

$$
z \equiv \exp \left(\frac{\mu}{T}\right)
$$

and then the above equations for the density and the pressure can be written as

$$
n=\frac{g}{2 \pi^{2}} \int_{0}^{\infty} \frac{p^{2}}{z^{-1} e^{\frac{p^{2}}{2 M_{\pi} T}}-1} d p
$$

and

$$
P=-\frac{g T}{2 \pi^{2}} \int_{0}^{\infty} p^{2} \ln \left(1-z e^{-p^{2} / 2 M_{\pi} T}\right) d p .
$$

Introducing the variable $x=p^{2} /\left(2 M_{\pi} T\right)$ we have

$$
n=\frac{g}{4 \pi^{2}}\left(2 M_{\pi} T\right)^{3 / 2} \int_{0}^{\infty} \frac{x^{1 / 2}}{z^{-1} e^{x}-1} d x
$$

and

$$
P=\frac{g}{6 \pi^{2}}\left(2 M_{\pi} T\right)^{3 / 2} T \int_{0}^{\infty} \frac{x^{3 / 2}}{z^{-1} e^{x}-1} d x
$$

where the integrals can be written in terms of the $g_{n}$ functions [9] as

$$
\int \frac{x^{n}}{z^{-1} e^{x}-1} d x=\Gamma(n+1) g_{n+1}(z)
$$

with

$$
g_{n}(z)=\sum_{l=1}^{\infty} \frac{z^{l}}{l^{n+1}}
$$

so that

$$
g_{s}(1)=\zeta_{s}
$$

and

$$
g_{s-1}(z)=z \frac{d g_{s}(z)}{d z}
$$

Therefore we have for a nonrelativistic free pion gas at equilibrium at low temperature and density

$$
n=g\left(\frac{M_{\pi} T}{2 \pi}\right)^{3 / 2} g_{3 / 2}(z)
$$

and

$$
P=g\left(\frac{M_{\pi} T}{2 \pi}\right)^{3 / 2} T g_{5 / 2}(z) .
$$

Thus the equation of state can be written as

$$
P=n T \frac{g_{5 / 2}(z)}{g_{3 / 2}(z)} .
$$

\section{THE KINETIC THEORY FOR PIONS}

The possibility of having a hydrodynamic description of the pion gas requires the definition of macroscopic fields such as the velocity $\vec{V}=\vec{V}(\vec{r}, t)$, the pressure $P=P(\vec{r}, t)$, the number density $n=n(\vec{r}, t)$, the temperature $T=T(\vec{r}, t)$, and others. In other words, it is needed to have a large number of pions in each volume element $d V$. The statistical description of the gas is based on the (one-particle) distribution function $f(\vec{r}, \vec{v}, t)$ which gives the number of pions inside the phasespace volume element $d \vec{r} d \vec{v}$ at the instant $t$. In fact the phase-space volume element is given by $d \vec{r} d \vec{p}$ but in the case considered here the difference amounts to a constant factor which we include in the definition of the distribution function $f$. Thus the total number of pions will be given by

$$
N=\int f(\vec{r}, \vec{v}, t) d \vec{r} d \vec{v}=\int n(\vec{r}, t) d \vec{r}
$$


As we are interested here in the behavior of the pion gas at low temperatures and densities, most of the pion interactions will occur at low energies. Then the pion-pion interactions will be essentially elastic and the total number of pions will be a conserved quantity. This in particular means that it makes sense to introduce the chemical potential $\mu=\mu(\vec{r}, t)$ related with the pion number even in the presence of lowenergy pion interactions. The evolution of the distribution function is determined by the BBKGY (Bogoliubov, Born, Kirkwood, Green, and Yvon) hierarchy [10]. This is a set of $N$ coupled equations for the $n$-particle distribution functions $f_{(n)}\left(\vec{r}_{1}, \vec{v}_{1}, \vec{r}_{2}, \vec{v}_{2}, \ldots, \vec{r}_{n}, \vec{v}_{n}, t\right)$ with $f=f_{(1)}$. However, for low-density gases it can be a good approximation to truncate the BBKGY hierarchy. For example, by using the Bogoliubov ansatz, i.e., by writing the two-particle function in terms of an appropriate product of two one-particle functions (molecular chaos hypothesis), it is possible to decouple the first BBKGY equation from the others to obtain the well known Boltzmann equation. This equation was modified by Uehling and Uhlenbeck in order to include the quantum statistic effects (see, for example, [11]). For the bosonic case the Uehling-Uhlenbeck equation reads

$$
\left(\partial_{t}+\vec{v}_{1} \vec{\nabla}_{\vec{r}}\right) f_{1}=C\left[f_{1}\right]
$$

where

$$
\begin{aligned}
C\left[f_{1}\right]= & \int d \vec{v}_{2} d \sigma u\left[f_{1}^{\prime} f_{2}^{\prime}\left(1+\frac{f_{1}}{A}\right)\left(1+\frac{f_{2}}{A}\right)\right. \\
& \left.-f_{1} f_{2}\left(1+\frac{f_{1}^{\prime}}{A}\right)\left(1+\frac{f_{2}^{\prime}}{A}\right)\right]
\end{aligned}
$$

is the collision term or collision functional. The above equation describes the irreversible space-time evolution of the (one-point) distribution function $f$, this irreversible behavior being a consequence of the truncation of the BBKGY hierarchy. As usual we use for short the notation $f_{i}=f\left(\vec{r}_{i}, \vec{v}_{i}, t\right)$ and $f_{i}^{\prime}=f\left(\vec{r}_{i}^{\prime}, \vec{v}_{i}^{\prime}, t\right)$ with $i=1,2 . d \sigma$ is the differential cross section corresponding to the elastic scattering of two pions with initial and final velocities $\vec{v}_{1}, \vec{v}_{2}$ and $\vec{v}_{1}^{\prime}, \vec{v}_{2}^{\prime}$, respectively. $u$ is the relative velocity of the initial particles $u$ $=\left|\vec{v}_{1}-\vec{v}_{2}\right|$ and, finally, the normalization constant $A$ is given by $A=g M_{\pi}^{3} /(2 \pi)^{3}$.

As it is the case of the Boltzmann equation, the UehlingUhlenbeck equation typically drives the gas from arbitrary initial distributions to the equilibrium distribution $f^{0}$ which is a fixed point of this equation in the sense that the collision functional vanishes on it:

$$
C\left[f^{0}\right]=0
$$

For a gas moving at a macroscopic velocity $\vec{V}$ the equilibrium distribution corresponding to temperature $T$ and pion chemical potential $\mu$ is given by

$$
f^{0}(\vec{r}, \vec{v}, t)=A\left(e^{1 / T\left(M_{\pi}(\vec{v}-\vec{V})^{2} / 2-\mu\right)}-1\right)^{-1}
$$

from which the properties of free pion gas given in the previous section can be derived. The case of local equilibrium is also described by this distribution but having the $\vec{V}, T$, and $\mu$ parameters depending on the position and time. This case corresponds to an ideal fluid. However, in order to describe dissipative processes, such as viscosity or thermal conduction, it is necessary to consider the case where the pion gas is not even in local thermal equilibrium.

\section{HYDRODYNAMIC EQUATIONS}

Given some magnitude $\psi$ it is possible to compute its statistical average by using the distribution function as

$$
\langle\psi\rangle=\frac{\int f \psi d \vec{r}}{\int f d \vec{r}}=\frac{\int f \psi d \vec{r}}{n(\vec{r}, t)},
$$

where $n=n(\vec{r}, t)$ is the pion number density. This average can be understood as the corresponding macroscopic magnitude in the hydrodynamic approach [12]. For example, the macroscopic fluid velocity $\vec{V}$ is given by

$$
\vec{V}=\frac{\int f \vec{v} d \vec{v}}{\int f d \vec{v}}=\frac{1}{n} \int f v d \vec{v}
$$

The equation governing the evolution of any of these magnitudes (transport equations) can be obtained by multiplying $\psi_{1}$ by the Uehling-Uhlenbeck equation and then integrating $\vec{v}_{1}$ so that

$$
\begin{aligned}
\int d & \vec{v}_{1}\left(\partial_{t}+\vec{v}_{1} \vec{\nabla}_{r}\right) f_{1} \psi_{1} \\
= & \int d \vec{v}_{1} d \vec{v}_{2} d \sigma u \psi_{1}\left[f_{1}^{\prime} f_{2}^{\prime}\left(1+A^{-1} f_{1}\right)\right. \\
& \left.\times\left(1+A^{-1} f_{2}\right)-f_{1} f_{2}\left(1+A^{-1} f_{1}^{\prime}\right)\left(1+A^{-1} f_{2}^{\prime}\right)\right] .
\end{aligned}
$$

The right-hand side term can be written in a more symmetric fashion as

$$
\begin{aligned}
& \frac{1}{4} \int d \vec{v}_{1} d \vec{v}_{2} d \vec{v}_{3} d \vec{v}_{4}|T|^{2}\left(\psi_{1}+\psi_{2}-\psi_{1}^{\prime}-\psi_{2}^{\prime}\right) \\
& \times\left[f_{1}^{\prime} f_{2}^{\prime}\left(1+A^{-1} f_{1}\right)\left(1+A^{-1} f_{2}\right)\right. \\
& \left.-f_{1} f_{2}\left(1+A^{-1} f_{1}^{\prime}\right)\left(1+A^{-1} f_{2}^{\prime}\right)\right],
\end{aligned}
$$

where $|T|^{2}$ is defined through the relation

$$
d \sigma=\frac{|T|^{2}}{\left|\vec{v}_{1}-\vec{v}_{2}\right|} d \vec{v}_{1}^{\prime} d \vec{v}_{2}^{\prime}
$$


From this form of the collision terms it becomes apparent than it vanishes for any quantity $\psi$ which is conserved in the collision such as the total energy or momentum. On the other hand, by using the definition of statistical average, the first term of Eq. (21) can be written as

$$
\partial_{t} n\langle\psi\rangle+\vec{\nabla}_{r} n\langle\psi \vec{v}\rangle .
$$

Thus for conserved magnitudes this term vanishes. In order to find the basic hydrodynamic equations it is useful to write the velocity as $\vec{v}=\vec{V}+\vec{\xi}$, i.e., $\vec{\xi}$ is the pion velocity relative to the macroscopic velocity $\vec{V}$ and therefore it averages to zero. Choosing $\psi=1, \vec{v}$, and $v^{2}$ (corresponding to the conservation of a constant, the momentum, and the energy in the elastic collision) we obtain the equations

$$
\begin{aligned}
\psi= & 1 \rightarrow \partial_{t} n+\vec{\nabla} n \vec{V}=0, \\
\psi= & \vec{v} \rightarrow \partial_{t} n V_{i}+\nabla_{j} n V_{i} V_{j}+\nabla_{j} n\left\langle\xi_{i} \xi_{j}\right\rangle=0, \\
\psi= & v^{2} \rightarrow \partial_{t} n\left(V^{2}+\left\langle\xi^{2}\right\rangle\right)+\nabla_{i} n V_{i}\left(V^{2}+\left\langle\xi^{2}\right\rangle\right) \\
& +\nabla_{i} 2 n V_{j}\left\langle\xi_{i} \xi_{j}\right\rangle+\nabla_{i} n\left\langle\xi_{i} \xi^{2}\right\rangle=0 .
\end{aligned}
$$

In order to understand the meaning of the above equations we can introduce the following macroscopic quantities:

$$
P_{i j}=M_{\pi} n\left\langle\xi_{i} \xi_{j}\right\rangle
$$

is the pressure tensor,

$$
Q=\frac{1}{2} M_{\pi} n\left\langle\xi^{2}\right\rangle
$$

is the internal energy density, and

$$
q_{i}=\frac{1}{2} M_{\pi} n\left\langle\xi_{i} \xi^{2}\right\rangle
$$

is the energy (heat) flux. Due to the isotropy of the pion fluid the pressure tensor can be taken as diagonal, i.e., $P_{i j}$ $=P \delta_{i j}$. In this case $Q=3 P / 2$ and $q_{i}=0$. Thus (by taking $\vec{V}=0$ but not its derivatives, which can always be done) we arrive to the standard ideal fluid equations, namely the continuity equation

$$
\partial_{t} n+n \nabla_{i} V_{i}=0
$$

the Euler equation

$$
n M_{\pi} \partial_{t} V_{i}+\nabla_{i} P=0
$$

and the energy conservation equation

$$
\partial_{t} Q+\frac{5}{3} Q \nabla_{i} V_{i}=0 \text {. }
$$

In spite of the above equations for the ideal fluid, it is well established that an irreversible flux of energy and momentum appear in an inhomogeneous gas. This flux gives rise to the well known dissipative processes of thermal conduction and viscosity. For example, a small gradient of temperature produces a heat flow which in a first approximation can be written as

$$
q_{i}=-\kappa \nabla_{i} T,
$$

where $\kappa$ is a coefficient called thermal conductivity. In order to include momentum diffusion effects we add an extra term to the isotropic pressure tensor:

$$
P_{i j}=P \delta_{i j}+P_{i j}^{\prime} .
$$

The requirement for this tensor to vanish for uniform translations and rotations of the fluid makes it possible to write it to first order in the velocity derivatives as [13]

$$
P_{i j}^{\prime}=-\eta\left(\frac{\partial V_{i}}{\partial x_{j}}+\frac{\partial V_{j}}{\partial x_{i}}-\frac{2}{3} \sum_{l=1}^{3} \frac{\partial V_{l}}{\partial x_{l}} \delta_{i j}\right)-\zeta \sum_{l=1}^{3} \frac{\partial V_{l}}{\partial x_{l}} \delta_{i j}
$$

Thus it is needed to introduce two coefficients $\eta$ and $\zeta$ which are usually called first and second viscosity coefficients. The introduction of the dissipative effects does not modify the continuity equation. However, the Euler equation becomes

$$
\begin{aligned}
\partial_{t} n V_{i} & +\nabla_{j} n V_{i} V_{j}+\frac{1}{M_{\pi}} \nabla_{j} P \delta_{i j} \\
= & \frac{1}{M_{\pi}} \nabla_{j}\left[\eta\left(\frac{\partial V_{i}}{\partial x_{j}}+\frac{\partial V_{j}}{\partial x_{i}}-\frac{2}{3} \sum_{l=1}^{3} \frac{\partial V_{l}}{\partial x_{l}} \delta_{i j}\right)\right. \\
& \left.+\zeta \sum_{l=1}^{3} \frac{\partial V_{l}}{\partial x_{l}} \delta_{i j}\right]
\end{aligned}
$$

which is known as the Navier-Stokes equation. The energy conservation equation is in this case

$$
\begin{aligned}
\partial_{t}\left(n V^{2}+\frac{2}{M_{\pi}} Q\right)+\nabla_{i} V_{i}\left(n V^{2}+\frac{2}{M_{\pi}} Q\right)+\nabla_{i} \frac{2}{M_{\pi}} V_{j} P \delta_{i j} \\
=\nabla_{i} \kappa \nabla_{i} T+\frac{2}{M_{\pi}} \nabla_{i} V_{j}\left[\eta\left(\frac{\partial V_{i}}{\partial x_{j}}+\frac{\partial V_{j}}{\partial x_{i}}-\frac{2}{3} \sum_{l=1}^{3} \frac{\partial V_{l}}{\partial x_{l}} \delta_{i j}\right)\right. \\
\left.+\zeta \sum_{l=1}^{3} \frac{\partial V_{l}}{\partial x_{l}} \delta_{i j}\right] .
\end{aligned}
$$

Thus the description of the dissipative flow of energy and momentum requires the introduction of three transport coefficients $\kappa, \eta$, and $\zeta$. In this work we will deal with the computation of the $\eta$ coefficient only (shear viscosity), but $\kappa$ and $\zeta$ can be computed in a similar way. In fact $\eta$ is the only relevant transport coefficient whenever heat conductivity can be neglected (adiabatic flow) and the fluid is incompressible. In principle any fluid can be considered as incompressible as far as the fluid velocity $V$ is small when compared with the fluid sound speed $v_{s}$ and no external fields are present. Therefore in the regime $V / v_{s} \ll 1$ it is always possible to neglect the second (bulk) viscosity $\zeta$. However the bulk viscosity must be taken into account in those circumstances 
where density cannot be taken as a constant. This is the case, for instance, of shock waves and other phenomena where the fluid velocity is comparable with the sound speed.

\section{COMPUTATION OF THE TRANSPORT COEFFICIENTS}

As it has been mentioned above the description of the dissipative processes requires going beyond local equilibrium. Thus in order to compute the transport coefficients we have to consider a distribution function $f$ slightly perturbed from the equilibrium distribution $f^{0}$, i.e.,

$$
f=f^{0}+\delta f=f^{0}+f^{0} \frac{\chi}{T},
$$

where $\chi$ is an arbitrary function of the velocities which represents the inhomogeneous contribution to the distribution function. This contribution will be assumed to be small in the sense that $\chi / T \ll 1$ since we are interested only in the computation of the transport coefficients. The $\chi$ function must by determined by solving the Uehling-Uhlenbeck equation which can be linearized with respect to $\chi$. Then it is possible to compute the transport coefficients from this function. For example, the heat flux can be written as

$$
q_{i}=\frac{1}{2} M_{\pi} \int f^{0}\left(1+\frac{\chi}{T}\right) v_{i} v^{2} d \vec{v}
$$

Taking into account that this flux vanishes for a homogeneous gas and using the definition of the heat conductivity we have

$$
-\kappa \nabla_{i} T=\frac{M_{\pi}}{2 T} \int f^{0} \chi v_{i} v^{2} d \vec{v} .
$$

Now it is useful to write the $\chi$ function as

$$
\chi=-\nabla_{i} T h_{i}(\vec{v})=-\nabla_{i} T v_{i} h(v) .
$$

Therefore

$$
\kappa \nabla_{i} T=\kappa_{i j} \nabla_{j} T
$$

with the tensor conductivity $\kappa_{i j}$ being given by

$$
\kappa_{i j} \equiv \frac{M_{\pi}}{2 T} \int f^{0} h v_{j} v_{i} v^{2} d \vec{v} .
$$

For an isotropic gas $\kappa_{i j}=\kappa \delta_{i j}$, so that we have the following equation for the heat conductivity:

$$
\kappa=\frac{M_{\pi}}{6 T} \int f^{0} h v^{4} d \vec{v}
$$

In a similar way it is possible to write the viscosity in terms of an $h$ function. In order to do that we write the pressure tensor in terms of the distribution function

$$
P_{i j}=M_{\pi} \int f^{0}\left(1+\frac{\chi}{T}\right) v_{i} v_{j} d \vec{v}
$$

For the present calculation it is enough to consider the case $\vec{V}=0$ (but not its derivatives). Then the nonisotropic component of the pressure tensor can be written as

$$
P_{i j}^{\prime}=\frac{M_{\pi}}{T} \int f^{0} \chi v_{i} v_{j} d \vec{v} .
$$

On the other hand this tensor can be written as

$$
P_{i j}^{\prime}=-2 \eta V_{i j}+\left(\frac{2}{3} \eta-\zeta\right) V_{h h} \delta_{i j},
$$

where we have introduced $V_{i j}=\left(\partial_{i} V_{j}+\partial_{j} V_{i}\right) / 2$. For further convenience we choose the perturbation to have the form

$$
\chi=V_{k l} h_{k l}(\vec{v})
$$

where $h_{k l}$ is a microscopic velocity dependent quantity which can be written as

$$
h_{k l}=\left(v_{k} v_{l}-\frac{1}{3} v^{2} \delta_{k l}\right) h(v) .
$$

Therefore by equating the two forms of $P_{i j}^{\prime}$ we find

$$
2 \eta V_{i j}=\eta_{i j k l} V_{k l}
$$

where as far as we are interested only in the first viscosity coefficient we have neglected the $\zeta$ contribution to $P_{i j}^{\prime}$, and $\eta_{i j k l}$ (the viscosity tensor) is given by

$$
\eta_{i j k l} \equiv \frac{M_{\pi}}{T} \int v_{i} v_{j}\left(v_{k} v_{l}-\frac{1}{3} v^{2} \delta_{k l}\right) f^{0}(v) h(v) d \vec{v}
$$

Due to several symmetries of this tensor it can be written as

$$
\eta_{i j k l}=\eta\left(\delta_{i k} \delta_{j l}+\delta_{i l} \delta_{j k}-\frac{2}{3} \delta_{i j} \delta_{k l}\right),
$$

so that we have

$$
\eta=\frac{M_{\pi}}{10 T} \int v_{i} v_{j}\left(v_{i} v_{j}-\frac{1}{3} v^{2} \delta_{i j}\right) f^{0}(v) h(v) d \vec{v} .
$$

As the $h$ function depends only on the velocity modulus it is possible to perform the angular integrations

$$
\eta=\frac{4 \pi}{15 T} A \int_{0}^{\infty} \frac{1}{z^{-1} \exp \left(\frac{M_{\pi} v^{2}}{2 T}\right)-1} v^{6} h(v) d \vec{v}
$$

In the following it will be useful to consider $h$ as a function of the adimensional variable $x$, i.e., $h=h(x)$ defined as

$$
x=\frac{M_{\pi} v^{2}}{2 T} \text {. }
$$

At this point it is customary to develop the $h(x)$ in terms of the Sonine polynomials. However, it is more appropriate in our case to introduce a new family of orthogonal polyno- 
mials $P_{r}^{s}(z ; x)$ since we are considering the UehlingUhlenbeck as the transport equation (i.e., we have taken into account quantum effects) instead of the more common Boltzmann equation. The definition and the main properties of these new polynomials can be found in the Appendix. In terms of $P_{r}^{s}$ the $h$ function can be written as

$$
h(x)=\sum_{s=0}^{\infty} B_{s} P_{r}^{s}(z ; x) .
$$

Therefore

$$
\eta=\frac{M_{\pi}}{10 T}\left(\frac{2 T}{M_{\pi}}\right)^{5 / 2} A \int_{0}^{\infty} d x \frac{x^{5 / 2}}{z^{-1} e^{x}-1} \sum_{s=0}^{\infty} B_{s} P_{r}^{s}(z ; x)
$$

and the distribution function can be written as

$$
f=f^{0}+\frac{f^{0}}{T} V_{i j}\left(v_{i} v_{j}-\frac{1}{3} \delta_{i j} v^{2}\right) \sum_{s=0}^{\infty} B_{s} P_{r}^{s}\left(z ; \frac{M_{\pi}}{2 T} v^{2}\right) .
$$

For all the families of polynomials, i.e., for all the $r$ values, we have exact conservation of the collision invariants. This fact makes it possible to choose $r$ so that we get the simplest integral. The appropriate value turns out to be $r$ $=5 / 2$ and then we get

$$
\eta=\frac{A \pi^{3 / 2}}{2}\left(\frac{2 T}{M_{\pi}}\right)^{5 / 2} g_{7 / 2}(z) B_{0} .
$$

In this way the computation of the viscosity of the pion gas has been reduced to the computation of the $B_{0}$ coefficient.

\section{SIMPLIFYING THE UEHLING-UHLENBECK EQUATION}

As discussed above the perturbed distribution function must be a solution of the Uehling-Uhlenbeck equation (15). In order to solve this equation it is quite convenient to write the left-hand side in terms of macroscopic quantities, as the temperature, velocity, density, and fugacity, and their derivatives. This can be done by using the equation of state and the hydrodynamic equations as follows. First of all at lowest order we have

$$
\begin{aligned}
\partial_{t} f+\vec{v} \vec{\nabla} f & =\partial_{t} f^{0}+\vec{v} \vec{\nabla} f^{0} \\
& =A \partial_{t}\left(\frac{1}{z^{-1} \exp \left(\frac{E}{T}\right)-1}\right) \\
& +A \vec{v} \vec{\nabla}\left(\frac{1}{z^{-1} \exp \left(\frac{E}{T}\right)-1}\right) .
\end{aligned}
$$

Thus the temporal derivative is

$$
\partial_{t} f^{0}=-\frac{f^{02} z^{-1} \exp \left(\frac{E}{T}\right)}{A}\left(\frac{1}{T} \partial_{t} E-\frac{E}{T^{2}} \partial_{t} T-\partial_{t} \log z\right),
$$

where the energy $E$ is given by

$$
E=\frac{M_{\pi}}{2}(\vec{v}-\vec{V})^{2}
$$

so that we have

$$
\partial_{t} f^{0}=\frac{f^{02}}{A z \exp \left(-\frac{E}{T}\right)}\left(\partial_{t} \log z+\frac{E}{T^{2}} \partial_{t} T+\frac{M_{\pi}}{T} v_{i} \partial_{t} V_{i}\right)
$$

In a similar way we can obtain

$$
v_{j} \nabla_{j} f^{0}=\frac{f^{02} v_{j}}{A z \exp \left(-\frac{E}{T}\right)}\left(\nabla_{j} \log z+\frac{E}{T^{2}} \nabla_{j} T+\frac{M_{\pi}}{T} v_{i} \nabla_{j} V_{i}\right)
$$

Thus we have

$$
\begin{aligned}
\partial_{t} f^{0}+v_{j} \nabla_{j} f^{0}= & \frac{f^{02}}{A z \exp \left(-\frac{E}{T}\right)}\left(\partial_{t} \log z+\frac{E}{T^{2}} \partial_{t} T\right. \\
& +\frac{M_{\pi}}{T} v_{i} \partial_{t} V_{i}+v_{j} \nabla_{j} \log z \\
& \left.+\frac{E}{T^{2}} v_{j} \nabla_{j} T+\frac{M_{\pi}}{T} v_{i} v_{j} V_{i j}\right) .
\end{aligned}
$$

Here it is possible to use the state equation for the free pion gas and the ideal fluid equations (Euler, continuity, and energy conservation) to find

$$
\begin{aligned}
\partial_{t} f^{0}+v_{j} \nabla_{j} f^{0}= & \frac{f^{02}}{A z \exp \left(-\frac{E}{T}\right)}\left[\frac{1}{T}\left(\frac{E}{T}-\frac{5}{2} \frac{g_{5 / 2}(z)}{g_{3 / 2}(z)}\right) v_{i} \nabla_{i} T\right. \\
& \left.+\left(\frac{M_{\pi}}{T} v_{i} v_{j}-\frac{2}{3} \frac{E}{T} \delta_{i j}\right) V_{i j}\right]
\end{aligned}
$$

On the other hand, substituting expression (37) into Eq. (16), expanding to first order in the perturbation $\chi$, and taking benefit from the equilibrium distribution function relation

$$
\begin{aligned}
f_{1}^{0 \prime} & f_{2}^{0 \prime}\left(1+A^{-1} f_{1}^{0}\right)\left(1+A^{-1} f_{2}^{0}\right) \\
\quad= & f_{1}^{0} f_{2}^{0}\left(1+f_{1}^{0 \prime}\right)\left(1+A^{-1} f_{2}^{0 \prime}\right)
\end{aligned}
$$

we obtain 


$$
\begin{aligned}
& f_{1}^{\prime} f_{2}^{\prime}\left(1+A^{-1} f_{1}\right)\left(1+A^{-1} f_{2}\right)-f_{1} f_{2}\left(1+A^{-1} f_{1}^{\prime}\right)\left(1+A^{-1} f_{2}^{\prime}\right) \\
& \quad=\frac{A^{2}}{T} \frac{z^{-2} e^{E / T}}{\left(z^{-1} e^{E_{1} / T}-1\right)\left(z^{-1} e^{E_{2} / T}-1\right)\left(z^{-1} e^{E_{1}^{\prime} / T}-1\right)\left(z^{-1} e^{E_{2}^{\prime} / T}-1\right)} \Delta\left\{\chi\left[1-z \exp \left(-\frac{E}{T}\right)\right]\right\},
\end{aligned}
$$

where $E=E_{1}+E_{2}=E_{1}^{\prime}+E_{2}^{\prime}$ and the $\Delta$ symbol is defined as

$$
\Delta[f(x)] \equiv f\left(x_{1}^{\prime}\right)+f\left(x_{2}^{\prime}\right)-f\left(x_{1}\right)-f\left(x_{2}\right) .
$$

Thus the whole collision term can be written as

$$
\begin{aligned}
C\left[f_{1}\right]= & \frac{1}{A^{2} T} \int d \vec{v}_{2} d \sigma u z^{-2} \exp (E / T) f_{1} f_{2} f_{1}^{\prime} f_{2}^{\prime} \Delta \\
& \times\left\{\chi\left[1-z \exp \left(-\frac{E}{T}\right)\right]\right\},
\end{aligned}
$$

where we have omitted the superindex 0 in the equilibrium distribution function. In this way the complete transport equation for the slightly unhomogeneous pion gas becomes

$$
\begin{aligned}
& \frac{f_{1}^{2}}{z e^{-M_{\pi} v_{1}^{2} / 2 T}}\left[\left(\frac{M_{\pi} v_{1}^{2}}{2 T}-\frac{5}{2} \frac{g_{5 / 2}(z)}{g_{3 / 2}(z)}\right) v_{1 i} \nabla_{i} T\right. \\
& \left.\quad+M_{\pi}\left(v_{1 i} v_{1 j}-\frac{1}{3} v_{1}^{2} \delta_{i j}\right) V_{i j}\right] \\
& =\frac{1}{A} \int d \vec{v}_{2} d \sigma u z^{-2} e^{M_{\pi} / 2 T\left(4 U^{2}+u^{2}\right)} \\
& \quad \times f_{1} f_{2} f_{1}^{\prime} f_{2}^{\prime} \Delta\left[\chi\left(1-z e^{-M_{\pi} v^{2} / 2 T}\right)\right],
\end{aligned}
$$

where we have defined $\vec{U}=\left(\vec{v}_{1}+\vec{v}_{2}\right) / 2=\left(\vec{v}_{1}^{\prime}+\vec{v}_{2}^{\prime}\right) / 2$, i.e., it is the collision center of mass velocity. Once again $\vec{u}=\vec{v}_{1}$ $-\vec{v}_{2}$ is the relative velocity of the incident pions. Thus, in particular, we have

$$
E=\frac{M_{\pi}}{2}\left(2 U^{2}+\frac{1}{2} u^{2}\right) .
$$

\section{LOW-ENERGY PION CROSS-SECTION}

In order to solve the above transport equation it is needed to know the differential cross section for pion scattering. As we are interested in the low energy regime the most appropriate approach is the chiral perturbation theory which relies on the chiral symmetry pattern of the strong interactions. In particular the chiral Lagrangian approach suggested by Weinberg is quite useful and extended up to the one loop level by Gasser and Leutwyler. This chiral Lagrangian approach consists of a systematic expansion on the pion field derivatives and the pion mass which are considered to be of the same order. From the lowest order of this expansion, the pion elastic scattering amplitude is given by

$$
\begin{aligned}
T_{a_{1}, a_{2}, a_{1}^{\prime}, a_{2}^{\prime}}(s, t, u)= & \frac{s-M_{\pi}^{2}}{F_{\pi}^{2}} \delta_{a_{1} a_{2}} \delta_{a_{1}^{\prime} a_{2}^{\prime}}+\frac{t-M_{\pi}^{2}}{F_{\pi}^{2}} \delta_{a_{1} a_{1}^{\prime}} \delta_{a_{2} a_{2}^{\prime}} \\
& +\frac{u-M_{\pi}^{2}}{F_{\pi}^{2}} \delta_{a_{1} a_{2}^{\prime}} \delta_{a_{2} a_{1}^{\prime}},
\end{aligned}
$$

where the standard Mandelstan variables $s, t$, and $u$ are related by $s+t+u=4 M_{\pi}^{2}$, the $a$ subindices refer to the pion isospin and $F_{\pi}$ is the pion decay constant. In the total isospin basis there are three independent amplitudes corresponding to $I=0,1,2$ which are given by

$$
\begin{aligned}
T^{0} & =\frac{2 s-M_{\pi}^{2}}{F_{\pi}^{2}}, \\
T^{1} & =\frac{t-u}{F_{\pi}^{2}}, \\
T^{2} & =\frac{2 M_{\pi}^{2}-s}{F_{\pi}^{2}} .
\end{aligned}
$$

The averaged modulo squared amplitude is given by

$$
|T|^{2}=\frac{1}{\sum_{I}(2 I+1)} \sum_{I}(2 I+1)\left|T^{I}\right|^{2}
$$

which leads to

$$
|T|^{2}=\frac{1}{9 F_{\pi}^{4}}\left[21 M_{\pi}^{4}+9 s^{2}-24 M_{\pi}^{2} s+3(t-u)^{2}\right] .
$$

The relativistic differential cross section for elastic pion scattering is

$$
d \sigma=\frac{|T|^{2}}{4 u E_{1} E_{2}} d \text { LIPS, }
$$

where the differential Lorentz invariant phase space is given by

$$
\begin{aligned}
d \text { LIPS } \equiv & C(2 \pi)^{4} \delta^{4}\left(p_{1}+p_{2}-p_{1}^{\prime}-p_{2}^{\prime}\right) \\
& \times \frac{d \vec{p}_{1}^{\prime}}{2(2 \pi)^{3} E_{1}^{\prime}} \frac{d \vec{p}_{2}^{\prime}}{2(2 \pi)^{3} E_{2}^{\prime}},
\end{aligned}
$$

where the constant $C=1 / 2$ takes into account the identity of the final pions. 
As discussed above we are interested in the nonrelativistic regime where $\vec{p}_{i}=M_{\pi} \vec{v}_{i}, \vec{P}=2 M_{\pi} \vec{U}$, and $E_{i}=M_{\pi}$ $+M_{\pi} v_{i}^{2} / 2$. In this limit it is convenient to write the cross section in terms of the center of mass and relative velocities $\vec{U}, \vec{u}$, and $\vec{u}^{\prime}$ defined as

$$
\vec{u}^{\prime}=\vec{v}_{1}^{\prime}-\vec{v}_{2}^{\prime}, \quad u=u^{\prime}
$$

After some standard calculations the nonrelativistic reduction of the cross section can be found to be

$$
\begin{aligned}
d \sigma= & \frac{23}{768} \frac{M_{\pi}^{2}}{\pi F_{\pi}^{4}}\left(1-\frac{3}{2} U^{2}+\frac{13}{184} u^{2}\right. \\
& \left.+U^{2} \cos \theta^{\prime} \frac{2 U+u \cos \theta^{\prime}}{2 U \cos \theta^{\prime}+u}\right) d \cos \theta^{\prime}
\end{aligned}
$$

where $\theta^{\prime}$ is the angle between by $\vec{U}$ and $\vec{u}^{\prime}$.

\section{LOWEST ORDER VISCOSITY FOR THE PION GAS}

As we have seen in previous sections the perturbation of the distribution function appropriate for the computation of the pion gas viscosity can be written as

$$
\chi=-V_{i j}\left(v_{i} v_{j}-\frac{1}{3} v^{2} \delta_{i j}\right) \sum_{s=0}^{\infty} B_{s} P_{5 / 2}^{s}(z ; x),
$$

where the $B_{s}$ coefficients must be obtained by solving the linearized transport equation, $B_{0}$ being the dominant one. In order to do so let us consider for a while a tensor $l_{k l}$ which is an arbitrary function on $z$ and $\hat{v}_{1}$. For further convenience we have defined the hat on any velocity as

$$
\hat{v}=\sqrt{\frac{M_{\pi}}{2 T}} v .
$$

By multiplying this tensor by the transport equation and integrating on $v_{1}$ we have

$$
\begin{aligned}
\int d \vec{v}_{1} l_{k l}\left(z ; \hat{v}_{1}\right) \frac{f_{1}^{2}}{z e^{-\hat{v}_{1}^{2}}}\left[\left(\hat{v}_{1}^{2}-\frac{5}{2} \frac{g_{5 / 2}(z)}{g_{3 / 2}(z)}\right) v_{1 i} \nabla_{i} T\right. \\
\left.+M_{\pi}\left(v_{1 i} v_{1 j}-\frac{1}{3} v_{1}^{2} \delta_{i j}\right) V_{i j}\right] \\
=-V_{i j} \sum_{s=0}^{\infty} B_{s} \frac{1}{A} \int d \vec{v}_{1} d \vec{v}_{2} d \sigma l_{k l}\left(z ; \hat{v}_{1}\right) u z^{-2} \\
\left.\quad \times e^{\left(2 \hat{U}^{2}+1 / 2 \hat{u}^{2}\right)} f_{1} f_{2} f_{1}^{\prime} f_{2}^{\prime} \Delta\right]\left(v_{i} v_{j}-\frac{1}{3} v^{2} \delta_{i j}\right) \\
\quad \times\left(1-z e^{\left.\left.-\hat{v}_{1}^{2}\right) P_{5 / 2}^{s}\left(z ; \hat{v}^{2}\right)\right] .}\right.
\end{aligned}
$$

Now we choose the tensor $l_{k l}$ to be

$$
l_{k l}(z ; \hat{v})=\left(v_{k} v_{l}-\frac{1}{3} v^{2} \delta_{k l}\right)\left(1-z e^{-\hat{v}^{2}}\right) P_{5 / 2}^{t}\left(z ; \hat{v}^{2}\right) .
$$

Then, one of the two integrals appearing in the right-hand side of the Eq. (82) vanishes, namely

$$
\int d \vec{v}_{1} l_{k l}\left(z ; \hat{v}_{1}\right) \frac{f_{1}^{2}}{z e^{-\hat{v}_{1}^{2}}}\left(\hat{v}_{1}^{2}-\frac{5}{2} \frac{g_{5 / 2}(z)}{g_{3 / 2}(z)}\right) v_{1 i} \nabla_{i} T=0 .
$$

Therefore the two sides of Eq. (82) are proportional to $V_{i j}$ which is arbitrary. Thus one possible solution to the equation is found by eliminating this tensor in both sides and then by contracting with the tensors $\delta_{i k} \delta_{j l}$ so that the right-hand side of Eq. (82) becomes

$$
\begin{gathered}
A^{2} V_{i j} \int d \vec{v} \frac{M_{\pi}\left(v_{k} v_{l}-\frac{1}{3} v^{2} \delta_{k l}\right)^{2}}{z^{-1} e^{\hat{v}^{2}}-1} P_{5 / 2}^{t}\left(z ; \hat{v}^{2}\right) \\
=V_{i j} A^{2} \frac{5 \pi^{3 / 2} M_{\pi}}{2}\left(\frac{2 T}{M_{\pi}}\right)^{7 / 2} g_{7 / 2}(z) \delta_{0 t} .
\end{gathered}
$$

In order to compute the $B_{s}$ coefficients we have to proceed in a similar way with the right-hand side of Eq. (82). For that purpose we will take the low energy limit of the differential cross section which is just

$$
d \sigma=\frac{23 M_{\pi}^{2}}{768 \pi F_{\pi}^{4}} d \cos \theta^{\prime}
$$

where $\theta^{\prime}$ belongs to the interval $(0, \pi)$. Therefore at very low energies the elastic pion scattering cross section can be considered as a constant, so that it is formally equivalent to the elastic scattering of rigid spheres of radius

$$
R=\frac{M_{\pi}}{\pi F_{\pi}^{2}} \sqrt{\frac{23}{384}}
$$

After the manipulations described below the right-hand side of the transport equation can be written as the collision term as

$$
V_{i j} \frac{23 M_{\pi}^{2} A^{3}}{768 F_{\pi}^{4}}\left(\frac{2 T}{M_{\pi}}\right)^{11 / 2} \sum_{s=0}^{\infty} B_{s} b_{s t}(z)
$$

where we have defined

$$
\begin{aligned}
b_{s t}(z)= & \int_{0}^{\infty} d U d u \int_{0}^{\pi} d \theta^{\prime} d \theta \int_{0}^{2 \pi} d \phi \sin \theta^{\prime} \sin \theta U^{2} u^{3} z^{-2} \\
& \times e^{2 U^{2}+1 / 2 u^{2}} \prod_{a_{1}, a_{2}, a_{1}^{\prime}, a_{2}^{\prime}}\left(z^{-1} e^{\left.v_{a}^{2}-1\right)^{-1}}\right. \\
& \times \Delta\left[\left(v_{k} v_{l}-\frac{1}{3} \delta_{k l} v^{2}\right)\left(1-z e^{-v^{2}}\right) P_{5 / 2}^{s}\left(z ; v^{2}\right)\right] \\
& \times \Delta\left[\left(v_{k} v_{l}-\frac{1}{3} \delta_{k l} v^{2}\right)\left(1-z e^{-v^{2}}\right) P_{5 / 2}^{t}\left(z ; v^{2}\right)\right] .
\end{aligned}
$$


Then the transport equation (82) can be written as

$$
\frac{23 M_{\pi} A}{1920 \pi^{3 / 2} F_{\pi}^{4}}\left(\frac{2 T}{M_{\pi}}\right)^{2} g_{7 / 2}^{-1}(z) \sum_{s=0}^{\infty} B_{s} b_{s t}=\delta_{0 t} .
$$

Therefore by defining

$$
\mathcal{B}_{s} \equiv \frac{23 M_{\pi} A}{1920 \pi^{3 / 2} F_{\pi}^{4}}\left(\frac{2 T}{M_{\pi}}\right)^{2} g_{7 / 2}^{-1}(z) B_{s}
$$

the viscosity is given by

$$
\eta=\sqrt{\frac{2 T}{M_{\pi}}} \frac{960 \pi^{3} F_{\pi}^{4}}{23 M_{\pi}} g_{7 / 2}^{2}(z) \mathcal{B}_{0},
$$

where $\mathcal{B}_{0}$ is a solution of the equation system

$$
\sum_{s=0}^{\infty} \mathcal{B}_{s} b_{t s}(z)=\delta_{t 0}
$$

or

$$
\left(\begin{array}{cccc}
b_{00}(z) & b_{01}(z) & b_{02}(z) & \cdots \\
b_{10}(z) & b_{11}(z) & b_{12}(z) & \cdots \\
b_{20}(z) & b_{21}(z) & b_{22}(z) & \cdots \\
\vdots & \vdots & \vdots & \ddots
\end{array}\right)\left(\begin{array}{c}
\mathcal{B}_{0} \\
\mathcal{B}_{1} \\
\mathcal{B}_{2} \\
\vdots
\end{array}\right)=\left(\begin{array}{c}
1 \\
0 \\
0 \\
\vdots
\end{array}\right)
$$

In order to find the leading contribution to the viscosity we can truncate the above system to find

$$
\mathcal{B}_{0}=\frac{1}{b_{00}(z)} .
$$

In this way the calculation of the viscosity has been reduced to the computation of the integral $b_{00}(z)$.

\section{RESULTS AND DISCUSSION}

According to our previous discussion the viscosity of the pion gas at low density and temperatures is given by Eq. (92), where $\mathcal{B}_{0}$ is the solution of the equation system given in Eq. (93) and the corresponding coefficients are defined in Eq. (89). Thus it is possible, at least in principle, to find the viscosity as a function of the temperature $T$ and the fugacity $z$ (or the pion chemical potential). Alternatively it is also possible to find the viscosity as a function of the temperature and the pion number density. Due to the complicated integrals appearing in the definition of the $b_{s t}(z)$ functions it has been possible to get numerical results only by Monte Carlo [14] integration. For the sake of simplicity most of these numerical results have been obtained by using the approximation of Eq. (95) and the hard sphere cross section of Eq. (86). In order to check the validity of these approximations we have computed the $4 \times 4$ matrix containing the first $b_{s t}(z)$ functions for the particular value $z=1$. The result and the corresponding errors is

$$
b_{s t}=\left(\begin{array}{cccc}
277.2 \pm 0.7 & -42 \pm 1 & -99 \pm 3 & -408 \pm 14 \\
-42 \pm 1 & 1043 \pm 8 & -395 \pm 8 & -800 \pm 30 \\
-99 \pm 3 & -395 \pm 8 & 10700 \pm 110 & -7100 \pm 200 \\
-408 \pm 14 & -800 \pm 30 & -7100 \pm 200 & 199000 \pm 2000
\end{array}\right)
$$

Now we consider the submatrices $1 \times 1,2 \times 2,3 \times 3$, and $4 \times 4$ and solve the corresponding system for the appropriate number of $\mathcal{B}$ so that we can check the convergence of the results as the size of the truncation is increased. The results are shown in Table I. There we see that the error is of the order of $1.6 \%$.

To take into account the velocity effects in the cross section we will consider Eq. (79) instead of Eq. (86) in the computation of the $b_{i j}$ integrals. Then we will have

$$
b_{s t}=b_{s t}^{0}+\frac{T}{M_{\pi}} b_{s t}^{1},
$$

where $b_{i j}^{0}$ is the leading term corresponding to the hard sphere approximation and

$$
\begin{aligned}
b_{00}^{1}= & \int_{0}^{\infty} d U d u \int_{0}^{\pi} d \theta d \alpha \int_{0}^{2 \pi} \\
& \times d \phi \frac{\sin \theta \sin \alpha U^{2} u^{3} e^{2 U^{2}+1 / 2 u^{2}}}{\left(e^{v_{1}^{\prime 2}}-1\right)\left(e^{v_{2}^{\prime 2}}-1\right)\left(e^{v_{1}^{2}}-1\right)\left(e^{v_{2}^{2}}-1\right)} \\
& \times\left(\frac{13}{92} u^{2}-3 U^{2}+2 U^{2} \cos \theta \frac{2 U+u \cos \theta}{2 U \cos \theta+u}\right) \\
& \times\left\{\Delta\left[\left(v_{i} v_{j}-\frac{1}{3} \delta_{i j} v^{2}\right)\left(1-e^{-v^{2}}\right)\right]\right\}^{2} .
\end{aligned}
$$

At the zero order in the sense of the previous table we have

$$
\mathcal{B}_{0}=\frac{1}{b_{00}}=\frac{1}{b_{00}^{0}+\frac{T}{M_{\pi}} b_{00}^{1}},
$$


TABLE I. Values for $\mathcal{B}_{i}$ for low $i$ in the hard spheres approximation, and the first orders in the approach explained in the text.

\begin{tabular}{lcccc}
\hline \hline & $\mathcal{B}_{0}$ & $\mathcal{B}_{1}$ & $\mathcal{B}_{2}$ & $\mathcal{B}_{3}$ \\
\hline Zero order & $3.608 \times 10^{-3}$ & & & \\
First order & $3.630 \times 10^{-3}$ & $1.497 \times 10^{-4}$ & & \\
Second order & $3.647 \times 10^{-3}$ & $1.654 \times 10^{-4}$ & $3.985 \times 10^{-5}$ & \\
Third order & $3.666 \times 10^{-3}$ & $1.765 \times 10^{-4}$ & $4.700 \times 10^{-5}$ & $9.902 \times 10^{-6}$ \\
\hline \hline
\end{tabular}

which at low temperatures can be written as

$$
\mathcal{B}_{0}=\frac{1}{b_{00}^{0}}\left(1-\frac{T}{M_{\pi}} \frac{b_{00}^{1}}{b_{00}^{0}}\right)
$$

so that

$$
\eta=\sqrt{\frac{2 T}{M_{\pi}}} \frac{960 \pi^{3} F_{\pi}^{4}}{23 M_{\pi}} g_{7 / 2}^{2}(z) \frac{1}{b_{00}^{0}}\left(1-\frac{T}{M_{\pi}} \frac{b_{00}^{1}}{b_{00}^{0}}\right) .
$$

This formula can be applied provided

$$
T \ll M_{\pi}\left|\frac{b_{00}^{0}}{b_{00}^{1}}\right| .
$$

After a numerical integration we find, for $z=1, b_{00}^{1}=$ $-310 \pm 40$. By comparison with our previous result $b_{00}^{0}$ $=277.2 \pm 0.7$ we get the upper bound for the temperature

$$
T \ll(0.90 \pm 0.12) M_{\pi},
$$

which means that the hard sphere approximation is safe provided the temperature is much smaller than the $90 \%$ of the pion mass. As we are working in a nonrelativistic framework this will always be the case and then no additional constraint on the applicability of our results is coming from the hard sphere approximation.

Therefore in the following we will work at zero order and in the hard sphere approximation. In this case the viscosity is given by

$$
\eta(T, z)=\sqrt{T} \frac{4096 \sqrt{2} \pi^{2} F_{\pi}^{4}}{345 M_{\pi}^{3 / 2}}\left[b_{00}(z)\right]^{-1}\left[\int_{0}^{\infty} \frac{x^{5 / 2}}{z^{-1} e^{x}-1} d x\right]^{2} .
$$

Some numerical results obtained from this formula are found in Table II for different values of the fugacity $z$. The classical case is just the limit of $z$ going to zero. This corresponds to a classical gas of hard spheres of radius

$$
R=\frac{M_{\pi}}{\pi F_{\pi}^{2}} \sqrt{\frac{23}{384}}
$$

Because of the particular way we have performed our computations the particular case $z=0$ is not numerically accessible. However, this case has been treated a long time ago and it is possible to find an analytic result for the viscosity, namely

$$
\eta=\sqrt{T} \frac{5 \sqrt{M_{\pi}}}{16 \sqrt{\pi} R^{2}}
$$

Thus we can check that our computations have the proper $z=0$ limit. In Fig. 1 we show the behavior of $b_{00}$ and the viscosity, for constant $T$, in terms of the fugacity. From these plots we learn that the viscosity of the bosonic quantum gas is smaller than that of the classical gas. This could be expected because of the following heuristic argument: After some elastic collision the emerging particles have more affinity for occupied states than classical ones. Therefore the microscopic transfer of momentum is more effective in a classical gas than in a bosonic gas and then the viscosity is also larger. A pictorial view of this fact is displayed in Fig. 2.

In many cases it is useful to have the viscosity as a function of the temperature and the density instead of the temperature and the fugacity. The pionic density can be written in terms of the temperature and the fugacity as

$$
\frac{n}{T^{3 / 2}}=\frac{g M_{\pi}^{3 / 2}}{\sqrt{2} \pi^{2}} \int_{0}^{\infty} \frac{x^{1 / 2}}{z^{-1} e^{x}-1} d x
$$

This equation also defines implicitly the fugacity as a function of the density and the temperature $z=z(n, T)$. However, due to the complexities of the above integral these functions have only been computed numerically. In Fig. 3 we plot $\eta / \sqrt{T}$ as a function of $n / T^{3 / 2}$. The computed points can be fitted quite well in the plotted range with a polynomial,

$$
f_{1}(x)=A+B x+C x^{2},
$$

with constants $A=0.1814 \pm 0.0004, B=(-2.42 \pm 0.03)$ $\times 10^{-4}$, and $C=(9.6 \pm 0.3) \times 10^{-8}$. Thus the function obtained (Fig. 3) for the viscosity versus the temperature and the density is

TABLE II. Viscosity values computed in different physical regimes.

\begin{tabular}{lc}
\hline \hline & $\eta\left(\mathrm{MeV}^{3}\right)$ \\
\hline Quantum case $(z=1)$ & $\sqrt{T} 4.00 \times 10^{5}$ \\
Quantum case $(z=0.05)$ & $\sqrt{T} 1.40 \times 10^{6}$ \\
Classical case & $\sqrt{T} 1.9 \times 10^{6}$ \\
\hline
\end{tabular}




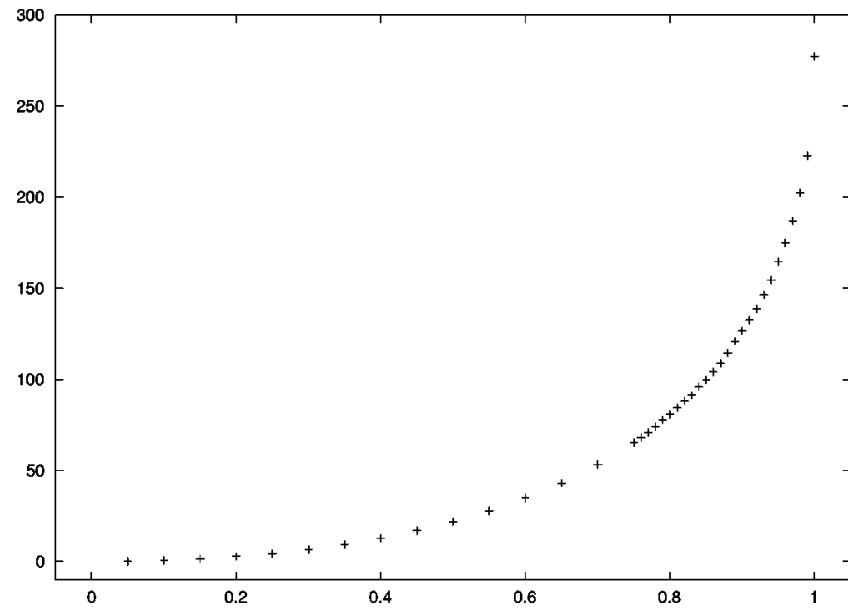

FIG. 1. Plot of $\left[b_{00}(z)\right]^{-1}\left[\int_{0}^{\infty} x^{5 / 2} / z^{-1} e^{x}-1 d x\right]^{2}$ versus $z$. It can be understood as a plot of the viscosity, for constant temperature and in arbitrary units, versus the fugacity.

$$
\begin{aligned}
\eta(T, n)= & \frac{4096 \sqrt{2} \pi^{2} F_{\pi}^{4}}{345 M_{\pi}^{3 / 2}} \sqrt{T}\left[0.1814-2.42 \times 10^{-4} \frac{n}{T^{3 / 2}}\right. \\
& \left.+9.6 \times 10^{-8}\left(\frac{n}{T^{3 / 2}}\right)^{2}\right] .
\end{aligned}
$$

As it may be noticed in Fig. 4, the values of the viscosity cover the range from $10^{6}$ to $10^{7} \mathrm{MeV}^{3}$ for the considered densities and temperatures.

\section{CONCLUSIONS}

In this work we have computed the viscosity of the pion gas, starting from first principles only. This computation is relevant for the hydrodynamic description of hadronic matter at low energies and densities. The work relies on the use of the Uehling-Uhlenbeck equation, which is the quantum version of the Boltzmann equation, and chiral symmetry, which
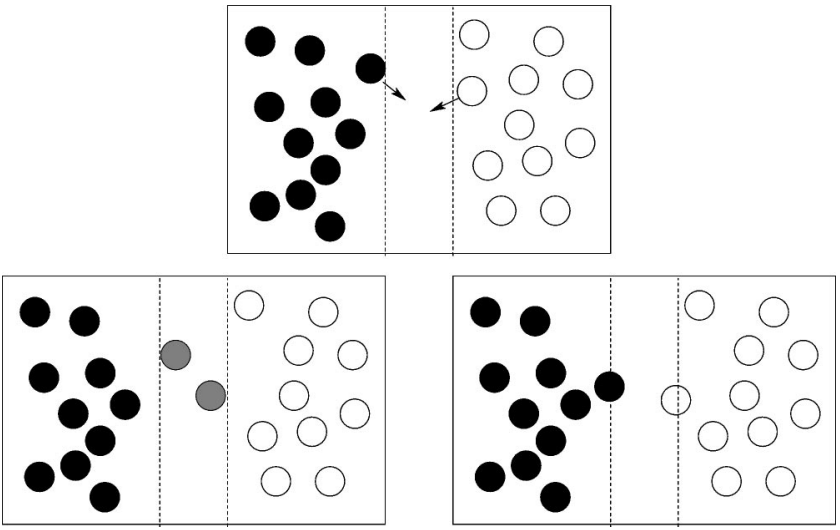

FIG. 2. (a) Two particles, belonging to two different regions of the gas with different average momentum (black and white), before an elastic collision. (b) Particles before the collision in the classical case where they typically get a new momentum (represented in gray). (c) Particles in the bosonic gas where they typically want to go to occupied states with the same momentum.

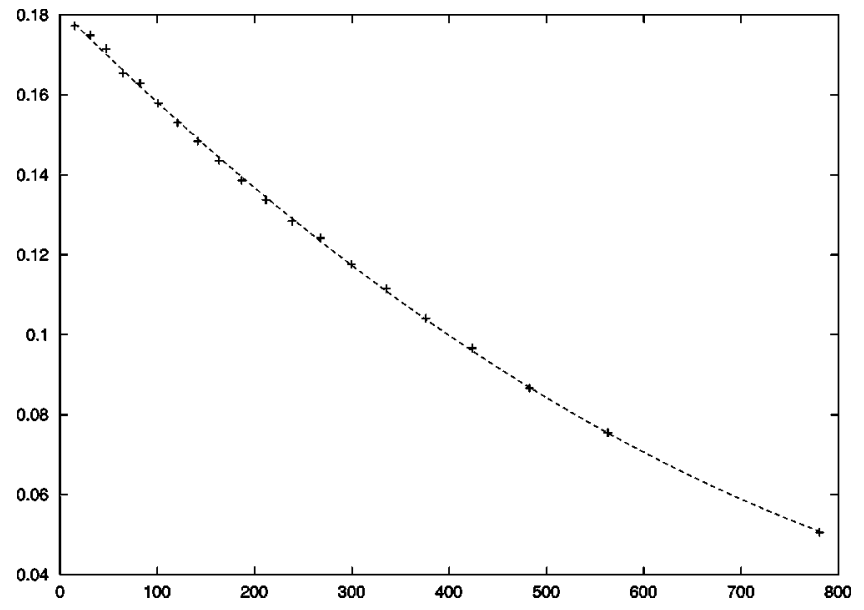

FIG. 3. Numerical fit of $\eta / \sqrt{T} \propto f_{1}\left(n / T^{3 / 2}\right)$.

determines completely the structure of the pion interactions at low energies. We have also found a formula for the viscosity in terms of the temperature and the density which properly fits our numerical results. The interest of the computations is twofold. First it is useful to check when the usual assumption of perfect fluid for the hadronic fluid is reasonable enough. Second it can be used to include the viscosity in the Navier-Stokes equations when dissipative effects cannot be neglected. The main limitation of our work is that it can be applied only in the nonrelativistic domain. As a consequence of that it cannot be used directly in the study of the events observed in the modern ultrarelativistic heavy ion colliders such as RHIC or the future LHC. In any case we understand that our work is interesting in order to show that the computations of transport coefficients of the pion gas can be done in a complete model independent way. In fact we consider that the result presented here is just the first step of a complete relativistic computation which could be applied at higher energies and therefore in more realistic situations, to improve the standard hydrodynamic description of had-

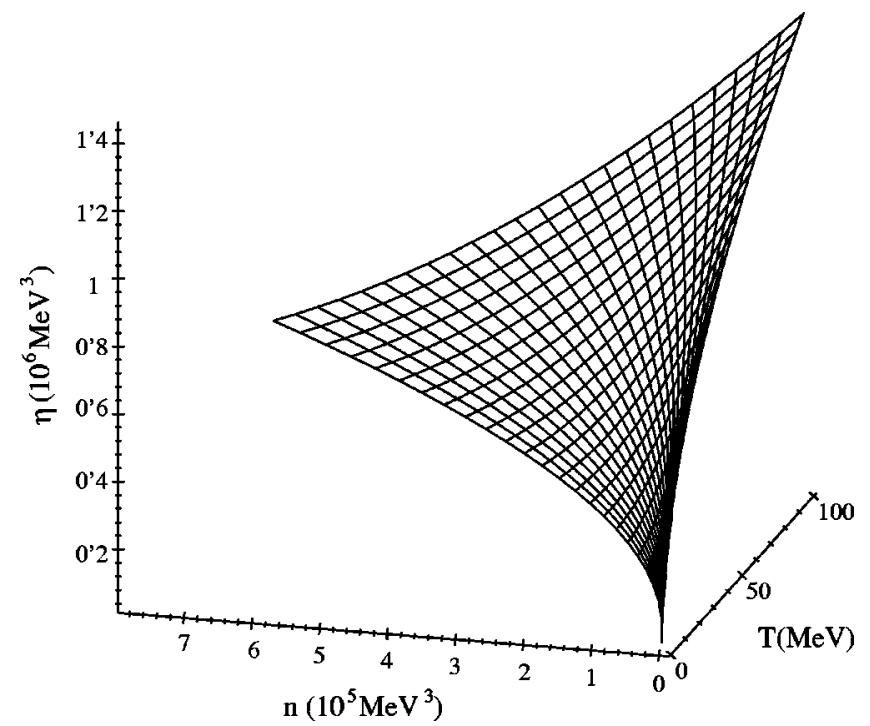

FIG. 4. 3D plot of viscosity vs temperature and particle number density, given by Eq. (109). 
ronic matter. Work is in progress in that direction.

\section{ACKNOWLEDGMENTS}

The authors want to thank A. Gomez Nicola and J. R. Pelaez for reading the manuscript. This work has been partially supported by the Ministerio de Educación y Ciencia (Spain) (CICYT AEN 97-1693 and PB98-0782).

\section{APPENDIX}

In this appendix we study the main properties of the family of orthogonal polynomials $P_{r}^{s}(z ; x)$ defined on the interval $(0, \infty)$ with measure

$$
d \mu_{r}(z ; x) \equiv \frac{x^{r}}{z^{-1} e^{x}-1}
$$

where $z \in(01]$ and $r>0$. By definition the polynomials are orthogonal for some given $r$, or in other words,

$$
\begin{aligned}
\left(P_{r}^{s}, P_{r}^{s^{\prime}}\right) & =\int_{0}^{\infty} d x \frac{x^{r}}{z^{-1} e^{x}-1} P_{r}^{s}(z ; x) P_{r}^{s^{\prime}}(z ; x) \\
& =A(z ; r, s) \delta_{s s^{\prime}} .
\end{aligned}
$$

For simplicity we define the polynomials so that they are monic, i.e., the coefficient of the term of highest degree in each polynomial is taken to be one. Thus the first polynomial is always the unity and its norm is given by

$$
P_{r}^{0}=1 \rightarrow\left(P_{r}^{0}, P_{r}^{0}\right)=\int_{0}^{\infty} d x \frac{x^{r}}{z^{-1} e^{x}-1}=\Gamma(r+1) g_{r+1}(z) .
$$

From the condition

$$
\left(P_{r}^{0}, P_{r}^{1}\right)=0
$$

it is possible to compute the second polynomial which turns out to be

$$
P_{r}^{1}(z ; x)=\frac{g_{r+2}(z)}{g_{r+1}(z)}(r+1)-x .
$$

The third polynomial is obtained from the conditions $\left(P_{r}^{0}, P_{r}^{2}\right)=0$ and $\left(P_{r}^{1}, P_{r}^{2}\right)=0$ which gives

$$
\begin{aligned}
P_{r}^{2}(z ; x)= & \frac{(r+3) g_{r+2}(z) g_{r+4}(z)-(r+2) g_{r+3}^{2}(z)}{(r+2) g_{r+1}(z) g_{r+3}(z)-(r+1) g_{r+2}^{2}(z)}(r+2)(r+1)+\frac{(r+3) g_{r+1}(z) g_{r+4}(z)-(r+1) g_{r+2}(z) g_{r+3}(z)}{(r+1) g_{r+2}^{2}(z)-(r+2) g_{r+1}(z) g_{r+3}(z)} \\
& \times(r+2) x+x^{2}
\end{aligned}
$$

Higher polynomials can be obtained in a similar way.

[1] J. D. Bjorken, Phys. Rev. D 27, 140 (1983).

[2] L. D. Landau, Izv. Akad. Nauk SSSR, Ser. Geogr. Geofiz. 17, 51 (1953).

[3] P. Gerber, H. Leutwyler, and J. L. Goity, Phys. Lett. B 246, 513 (1990).

[4] S. Weinberg, Physica A 96, 327 (1979); J. Gasser and H. Leutwyler, Ann. Phys. (N.Y.) 158, 142 (1984).

[5] E. M. Lifshitz and L. P. Pitaevskii, Kinetic Theory (Pergamon, New York, 1981), Vol. 10.

[6] M. Prakash, M. Prakash, R. Venugopalan, and G. Welke, Phys. Rep. 227, 321 (1993).

[7] D. J. Amit and Y. Verbin, Statistical Physics, An Introductory Course (World Scientific, Singapore, 2000).
[8] A. Dobado and J. R. Peláez, Phys. Rev. D 59, 034004 (1999).

[9] M. Abramowitz and I. A. Stegun, Handbook of Mathematical Functions (Dover, New York, 1968).

[10] R. L. Liboff, Kinetic Theory, Classical, Quantum and Relativistic Descriptions (Prentice-Hall, Englewood Cliffs, NJ, 1999).

[11] E. A. Uehling and G. E. Uhlenbeck, Phys. Rev. 43, 552 (1933).

[12] R. Balescu, Equilibrium and Nonequilibrium Statistical Mechanics (Wiley, New York, 1975).

[13] L. Landau and E. M. Lifshitz, Fluid Mechanics (Pergamon, New York, 1981), Vol. 6.

[14] W. H. Press et al., Numerical Recipes in C (Cambridge University Press, Cambridge, England, 1997). 